\title{
A Systems Modeling Methodology For Evaluation of Vehicle Aggressivity In The Automotive Accident Environment
}

\author{
Alexandra C. Kuchar \\ Volpe National Transportation Systems Center \\ Robert Greif \\ Tufts University \\ George W. Neat \\ Volpe National Transportation Systems Center
}

\begin{abstract}
A systems modeling approach is presented for assessment of harm in the automotive accident environment. The methodology is presented in general form and then applied to evaluate vehicle aggressivity in frontal crashes. The methodology consists of parametric simulation of several controlled accident variables, with case results weighted by the relative frequency of each specific event. A hierarchy of models is proposed, consisting of a statistical model to define the accident environment and assign weighting factors for each crash situation case, and vehicle and occupant models for kinematic simulation of crash events. Head and chest injury results obtained from simulation are converted to harm vectors, in terms of probabilistic Abbreviated Injury Scale (AIS) distributions based on previously defined risk analyses. These harm vectors are weighted by each case's probability as defined by the statistical model, and summed to obtain a total estimate of harm for the accident environment. The methodology is applied to a subset accident environment consisting of single- and two-vehicle frontal collisions among passenger cars and light trucks. The model is validated against recent crash statistics, and is found to accurately reflect trends in distribution of injury severity while slightly underestimating moderate to severe injuries. The model is subsequently exercised for variable sensitivity analyses, wherein the effects of light truck/car population mix are evaluated in terms of their impact on occupant harm within the subset accident environment.
\end{abstract}

\subsection{Introduction}

This paper presents a systems modeling approach for evaluation of overall safety in the automotive fleet. This methodology stands in contrast to typical approaches, where specific safety issues such as air bags are addressed independently. However, the recent surge in light truck sales in the U.S. has led to the advent of a broader problem: how to evaluate the aggressivity of these large heavy vehicles in twovehicle accidents while also considering their potential safety benefits in single-vehicle crashes. While light truck vehicles do provide added protection to occupants within the vehicle, one recent statistical study reports that light trucks are so aggressive due to both mass and geometry that in head-on crashes between cars and light trucks, deaths in the cars outnumber those in the light trucks by $70 \%$ (Joksch, 1998). The systems model methodology applied here features computational vehicle models to represent cars and light trucks, making it suitable for analysis of aggressivity and compatibility among dissimilar vehicles.

This paper describes a systems modeling methodology for prediction of passenger injuries across the entire accident environment, considering a variety of metrics including vehicle type, impact speed, occupant size, safety belt usage, and other factors which directly affect overall safety. This approach will allow for evaluation of global effects of small changes to the accident environment, so that proposed automotive safety regulations may be evaluated in terms of their total safety benefit. The methodology has been developed as a generalized tool for assessment of a variety of crashworthiness topics, such as air bags and vehicle design characteristics. The methodology is applied here to study vehicle aggressivity in terms of the relationship between passenger vehicle fleet mix and overall harm.

History. Several previous studies have considered a systems approach for investigating vehicle safety. During 1975-78, the Ford Motor Company developed the Safety Systems Optimization Model (Ford Motor Co., 1978), featuring a simulation-optimization program for maximizing a single vehicle's safety performance in frontal crashes. The same program was substantially modified by the University of Virginia in the early 1980s (White, et al., 1985), to include 


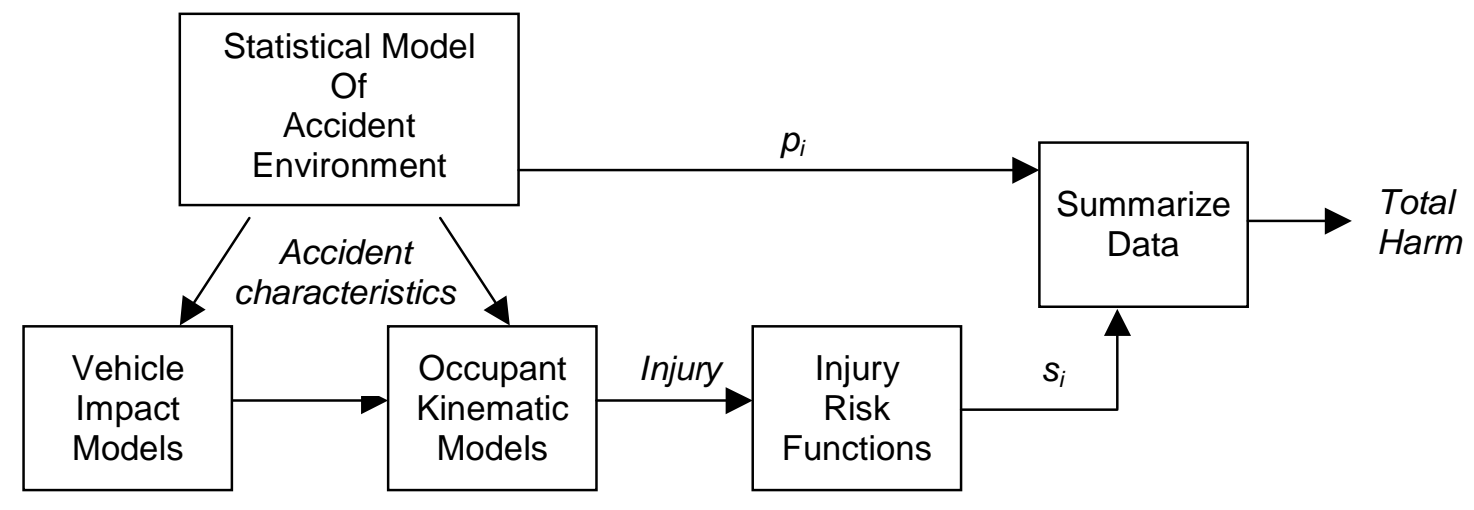

Figure 1. Fleet Systems Model Methodology

new biomechanical transforms and updated accident data as well as multivariate analysis capability. This model utilized approximating functions to estimate relationships between crash variables due to limitations in computational power at the time. Other motor vehicle manufacturers, including Fiat and Volkswagen, have also developed programs for optimizing vehicle design for crashworthiness, with emphasis on single-vehicle as opposed to fleet wide performance.

The model presented here differs from these earlier models in several aspects. It predicts total harm over a range of vehicle types rather than a single subject vehicle. While the model estimates injuries over a given set of crashes, it does not include an optimization algorithm for minimization of total harm. The model considers air bags in addition to seat belts, and occupants of varying size. It also incorporates recent accident statistics and more sophisticated biomechanical transforms than earlier approaches. Furthermore, due to improvements in structural modeling techniques and computer efficiency, the model includes parametric simulation of a range of vehicle and occupant crashworthiness models.

Governing Equation and Methodology. The methodology is based upon the following governing equation for estimation of total injuries:

$$
\text { Harm }=\sum_{i} p_{i} S_{i}
$$

Each $i$ is a specific crash event, defined in terms of assigned values for model variables, such as vehicle type, restraint usage and occupant size. The accident environment is described by the range of $i$, which may include as few or as many cases as desired. $p_{i}$ is the probability of each event $i$, its expected rate of occurrence based on accident statistics. $s_{i}$ is the expected injury outcome of crash event $i$, represented in terms of probable levels of harm as measured by the Abbreviated Injury Scale (AIS). Each case's expected harm outcome $s_{i}$ is determined via computer simulation of vehicle crashes and occupant kinematics. This formulation allows for consideration of a range of accidents, while assigning an appropriate weight to each event based upon field data. Given this methodology, the model's robustness is directly related to all three of these factors: the number and range of accidents considered, the reliability of the accident field data, and the accuracy of the computational models.

Figure 1 graphically depicts the implementation of the methodology. The term "Fleet Systems Model" refers to the whole system, which consists of a family of models. There are statistical models to describe the accident environment, vehicle structural crashworthiness models to predict vehicle behavior, occupant kinematic models to simulate dummy motion, and injury risk functions for estimation of harm.

\subsection{Statistical Model of Accident Environment}

The motive for examination of accident field data for development of a statistical model is threefold: to select and define the boundaries of the model environment, for case weighting (computation of $p_{i}$ for each case), and to provide a set of validation data against which the model's estimates of total injury are compared.

Subset Environment. A review of past year crash statistics is performed to identify the boundaries of the model environment - the subset of the real crash environment to be represented within the model. By identifying those events that are most frequent and lead to the greatest number of injuries, the model's coverage of the real environment can be maximized for a given number of cases.

The annual distribution of passenger vehicles in singleand two-vehicle towed accidents by impact mode and severity outcome is given in Table 1. Severe crashes are defined as those in which at least one occupant sustains an injury of AIS 3 or higher.

Note that vehicles in frontal accidents comprise a large percentage of severe crashes: $15.2 \%$ of these vehicles are in frontal single-vehicle impacts, while $10.7 \%$ are in twovehicle head-on accidents for a total of $25.9 \%$ of all vehicles in towed accidents. Side impacts are more common, accounting for $46.7 \%$ of vehicles in all crashes and $33.0 \%$ of 
Table 1. Vehicles in Towed Crashes, by Accident Mode, 1992-97'

\begin{tabular}{|l|c|c|}
\hline Crash Mode & $\begin{array}{c}\text { All Crashes } \\
(\mathbf{n}=\mathbf{3 . 4 5} \text { million } \\
\text { vehicles/year })\end{array}$ & $\begin{array}{c}\text { Severe Crashes } \\
(\mathbf{n}=\mathbf{9 3 , 0 0 0} \\
\text { vehicles/year })\end{array}$ \\
\hline $\begin{array}{c}\text { Single Vehicle } \\
\text { Fixed Object, } \\
\text { Frontal } \\
\text { Rollover }\end{array}$ & $6.7 \%$ & $15.2 \%$ \\
Other & $5.7 \%$ & $17.9 \%$ \\
\hline Two Vehicle & $11.7 \%$ & $12.1 \%$ \\
Head-on & $3.0 \%$ & $10.7 \%$ \\
Side Impact & $46.7 \%$ & $33.0 \%$ \\
Rear & $18.8 \%$ & $4.9 \%$ \\
Sideswipe & $5.0 \%$ & $4.5 \%$ \\
Other & $2.4 \%$ & $1.6 \%$ \\
\hline
\end{tabular}

vehicles in severe crashes. Single-vehicle rollover accidents also comprise a large percentage of vehicles in severe crashes, at $17.9 \%$. Because current frontal vehicle crashworthiness models are more feasible for parametric simulation than side impact models, the application of the methodology presented here considers only frontal impacts. This includes all single- and two-vehicle frontal impacts among cars and light trucks. Furthermore, while the vehicle crashworthiness models employed here simulate full frontal impacts, they are also assumed to approximate angled and offset crashes.

The same methodology is applied to select other parameters of the subset crash environment, wherein emphasis is placed on the frequency and severity outcome of impact variables, as well as modeling feasibility of impact parameters. For example, vehicle type is limited to passenger vehicles under gross vehicle weight of $4550 \mathrm{~kg}(10,000 \mathrm{lbs}$.), while all other vehicle groups such as buses and motorcycles are excluded. The passenger vehicle population is modeled as two separate classes, cars and LTVs (including all light trucks, sport utility vehicles, vans and minivans). All vehicles are assumed to be equipped with air bags, given the current trend towards an all-air bag fleet as older cars are retired from the road. It is recognized that modeling a fully air bag equipped fleet is a significant assumption, given that more than half of currently registered passenger vehicles in the U.S. are not equipped with air bags. However, this study is intended to serve as an initial test case for this systems modeling methodology. It is believed that this air bag assumption will likely lead to underestimation of severe occupant injuries to some degree. Future refinements to the model will include improved estimation of injuries in non-air bag equipped vehicles, either via simulation of non-airbag equipped vehicle interior models, or approximating functions. Occupant seat position is limited to front seat driver and passenger occupants only, as this group represents over $86 \%$

${ }^{1}$ All accident field data presented are obtained from the NHTSA National Automotive Sampling System (NASS) Crashworthiness Data System (CDS) database, years 199297. of all occupants in towed crashes. For estimation of harm in each simulated case, only injuries to the head and chest are considered, as these body areas are by far the most common region of serious injury in frontal collisions. Furthermore, the computational occupant models utilized for this application are validated only for head and chest response.

Given this parametric definition of the subset environment, it represents a total of 335,000 vehicles in towed accidents per year, 24,000 of which feature an occupant sustaining a severe injury.

Computation of Case Weights $p_{i}$. The $p_{i}$ term of the governing equation is a function of several accident variables:

$$
\begin{aligned}
& p_{i}=f n \text { (mode, vehicle, speed, belt usage, seat position, } \\
& \text { occupant size) }
\end{aligned}
$$

Each unique permutation of these variables defines a single case within the methodology, and the sum of all of these cases describes the entire subset environment considered. For each variable, the relative probability of each value is determined from field data. Some interdependencies exist among these six variables, as illustrated in Figure 2. The first and only probabilistically independent simulation parameter is accident mode, which determines the relative probability of each vehicle type. Both impact mode and vehicle type determine weighting of each simulated vehicle impact speed. Vehicle type alone determines occupant seat distribution, which in turn defines the probabilities of the occupant size and seat belt usage variables. The numbers in parenthesis in Figure 2 indicate the number of permutations for each variable. This yields a total of 504 cases, obtained via perturbation of 2 vehicle types, 2 impact modes (singleand two-vehicle impacts), 2 partner vehicles (car and LTV) in one of the impact modes (two-vehicle impacts), 7 impact speeds, 3 occupant sizes, 2 occupant locations, and 2 belt configurations.

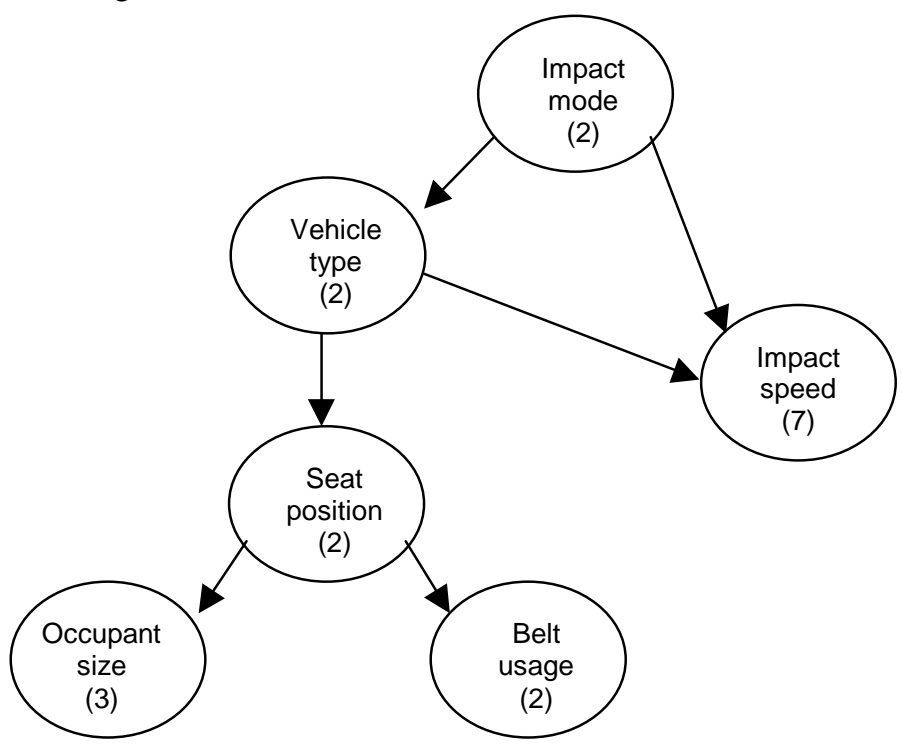

Figure 2. Hierarchy of Dependencies Among Probabilities of Simulation Variables 
For variables with discrete values (such as seat belt usage, yes or no), linked probabilities are derived directly from field data. For continuous variables such as vehicle speed and occupant size, probability density functions (PDFs) are defined, and relative probabilities of each value are computed via integration. Impact speeds for case simulation are selected to emphasize severe accidents, but are weighted according to frequency across all accidents.

The statistical model of the subset environment provides the data for computation of $p_{i}$ for each case in terms of the linked probabilities depicted in Figure 2.

$$
\begin{gathered}
p_{i}=p(\text { mode }) \times p(\text { vehicle } \mid \text { mode }) \times p(\text { speed } \mid \text { vehicle }, \text { mode }) \times \\
p(\text { seat position } \mid \text { vehicle }) \times p(\text { occupant size } \mid \text { seat position }) \\
\quad x p(\text { belt usage } \mid \text { seat position })
\end{gathered}
$$

Note that the computed value of $p_{i}$ for each case should be very small, given that there are a total of 504 cases evaluated for the subset environment, and the sum of all $p_{i}$ must equal 1.

\subsection{Vehicle Crashworthiness Simulation Models}

Vehicle response for each case is simulated using computational models of vehicles in frontal impacts. The vehicle models are one dimensional lumped-parameter systems, with three discrete masses representing the Occupant Compartment, Engine, and Wheels. Six non-linear springs represent energy-absorbing load paths in the front end of the vehicle, and are defined to approximate various buckling and crushing modes during frontal impacts. Each spring is described in terms of a segmented force-deflection curve to represent static behavior, plus a dynamic magnifier component, which applies dynamic force as a function of the spring's strain rate and static characteristics.

The models are simulated and developed using the SISAME (Structural Impact Simulation And Model Extraction) program (Mentzer, 1999), which performs forward simulation of one-dimensional lumped-parameter models, and features an optimization tool for the development of these models. This model extractionoptimization approach has been proven for development of highly accurate 1-dimensional models of vehicles in full frontal impacts (Mentzer et al., 1992). More recent developments in the SISAME program enable multiple-event extraction of simulation models, where crash test data from more than one event may be used to identify an optimal simulation model.

The passenger car fleet is represented by a single vehicle model based on a 1995 Chevrolet Lumina and the LTV fleet is represented by a model of a 1995 Ford Explorer. Future development of the methodology will include additional vehicle models to represent multiple weight classes of cars and LTVs, as well as more geometrically detailed vehicle models to capture $3 \mathrm{D}$ effects such as bumper mismatch. However, for initial validation of this systems approach, the 1-dimensional full frontal crashworthiness models are considered adequate for this application. Each model is extracted from two full frontal crash tests conducted at different speeds (24 and $56 \mathrm{kph}$ for the car, and 48 and 56 $\mathrm{kph}$ for the LTV). Both models demonstrate very good correlation with test data at both impact speeds. No test data was available for validation of the models in vehicle-tovehicle impacts.

The models are simulated in single- and two-vehicle full frontal impacts at 7 different impact speeds to generate occupant compartment response data for input into occupant simulation models.

\subsection{Occupant Models}

Two occupant kinematic models of a car and LTV (also based on a Chevrolet Lumina and Ford Explorer, respectively) were developed and simulated to generate head and chest injury measurements for the subset accident environment. The models are simulated using the MADYMO (Mathematical Dynamic Model) program, commonly used for vehicle crashworthiness applications. Each vehicle interior model is characterized by vehicle interior surfaces defined by planes with known forcedeflection characteristics under impact, and the air bags are modeled using finite elements. Each model is validated for head and chest response against a frontal 56kph (35mph) rigid barrier crash test. They are subsequently simulated for all of the vehicle crash pulses generated by the vehicle models, and varied with regard to occupant size $\left(5^{\text {th }}\right.$ percentile female, $50^{\text {th }}$ percentile male, and $95^{\text {th }}$ percentile male), occupant location (driver or passenger), and seat belt usage.

\subsection{Biomechanical Models}

Because the vast majority of serious injuries in crashes are the result of head and chest trauma, only head and chest injury metrics are used here to measure occupant harm. Further refinement of the methodology may consider other injury mechanisms, such as neck and femur loads.

Head Injury Criterion (HIC) is computed from triaxial head acceleration response from each occupant simulation case. Chest injury for each simulation case is measured in terms of the Combined Thoracic Index (CTI), defined as

$$
C T I=\frac{A_{\max }}{A_{\mathrm{int}}}+\frac{D_{\max }}{D_{\mathrm{int}}}
$$

where $A_{\max }$ and $D_{\max }$ are peak values observed during simulation and $A_{i n t}$ and $D_{i n t}$ are constants defined for each dummy size. CTI is not currently used as a regulatory criterion, though it is recommended by the National Highway Traffic Safety Administration (NHTSA) for research use (Kleinberger, et al., 1998).

Because both HIC and CTI are computed from a controlled environment - direct measurements of acceleration, deflection, or force during crash tests or computer simulation - they are not obtainable from field data. Injuries in real crashes are recorded in terms of the Abbreviated Injury Scale (AIS), which is less precise than the standard injury criteria. Nonetheless, the AIS scale is the only source of injury data available from the field, and 
provides the validation data for comparison of injury results computed by the Fleet Systems Model.

A series of mathematical models to convert HIC and CTI into the AIS scale are presented in Figures 3 and 4, commonly known as injury risk functions (Eppinger et al., 1999). These functions are proposed by NHTSA based on experimental data and previous research to estimate harm from measured criteria. The HIC injury risk functions are log normal approximations, while the CTI curves are twoparameter Weibull approximations. Both sets of curves are based upon experimental tests within the regulatory range of interest $\left(\mathrm{HIC}=1000, A_{\max }=60 \mathrm{G}\right.$ and $\left.D_{\max }=76 \mathrm{~mm}\right)$, and therefore these approximations are more heuristic for higher injuries. The AIS=6 curve shown below for CTI is not proposed by NHTSA, but has been extrapolated for use within this study.

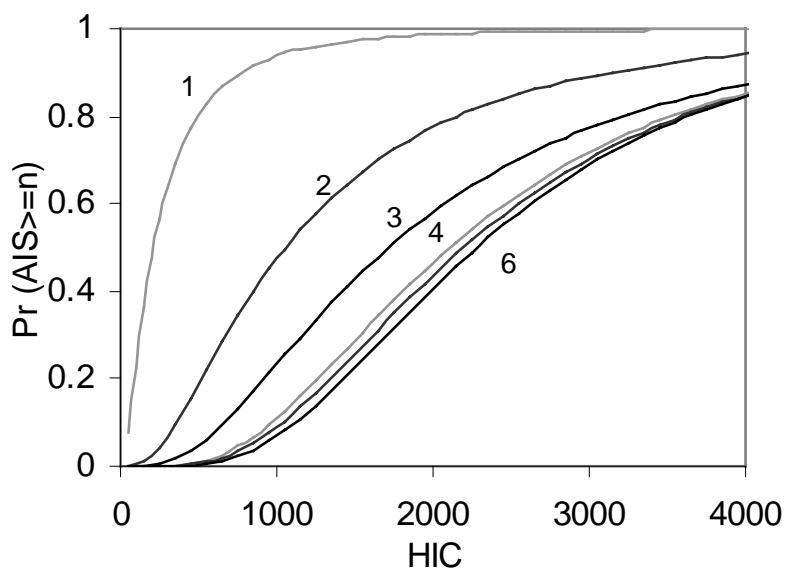

Figure 3. Injury Risk Functions for HIC (from Eppinger, et al., 1999)

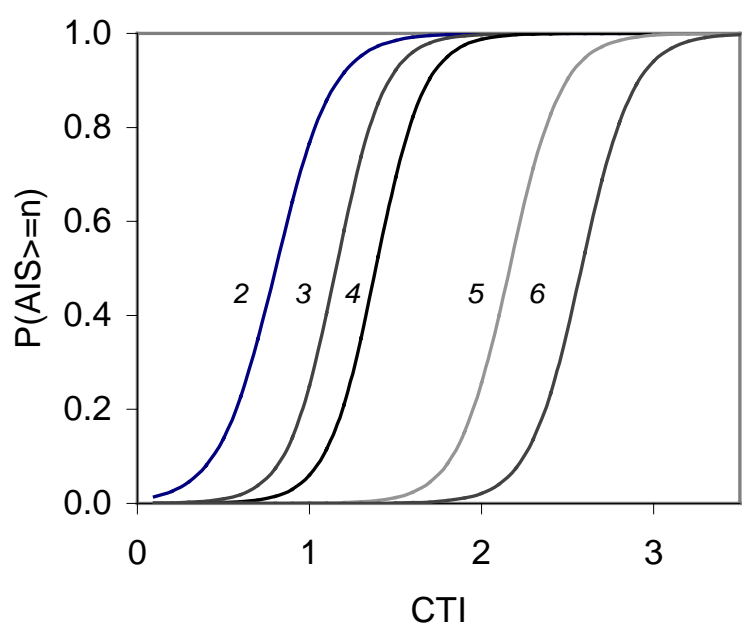

Figure 4. Injury Risk Functions for CTI (from Eppinger, et al., 1999)

Applying these functions provides a mathematical transform for conversion of HIC or CTI into probabilities of each AIS result. Hence, the injury risk functions can be applied to obtain the probabilities of AIS=0,1,2,3,4,5, and 6 .
Because these are cumulative probabilities, each AIS probability is subtracted from the next AIS $n+1$ probability to obtain a vector of probabilities summing up to 1.0. These values correspond to a vertical "slice" through Figure 3 or 4 at a given injury value. For each HIC and CTI computed from simulation, a vector of AIS probabilities is computed, corresponding to that occupant's probability of sustaining head or chest injuries corresponding to each AIS state. There are therefore two harm vectors obtained from each occupant simulation, one for head injuries (HIC) and the other for chest injuries (CTI). For each occupant simulation, the head and chest harm vectors are each multiplied by a normalized cost function, which quantifies the relative harm of each AIS level (NHTSA, 1999). The vector resulting in greater computed harm is assigned to be the vector $s_{i}$, or probabilistic AIS outcome, for that simulation case.

\subsection{Fleet Systems Model Results}

Parametric simulation of the vehicle and occupant models yields a total of 504 individual cases. For each of these cases there is a probability value $\left(p_{i}\right)$ obtained from the statistical model and a harm vector $\left(s_{i}\right)$ obtained from vehicle, occupant, and biomechanical models. These quantities are multiplied and summed according to the governing equation (1) to yield an estimated distribution of injuries for the subset environment.

The computed AIS distribution for the entire subset environment is shown in Figure 5, compared against field data. Because non-injuries and minor injuries of AIS 0,1, and 2 comprise the vast majority of the results, and because severe injuries of AIS 3 and higher are of greatest interest, only serious injuries are plotted, with minor injury figures given in text. A total annual number of 384,000 occupants is represented, so $1 \%$ of the environment corresponds to roughly 3,840 occupant injuries.

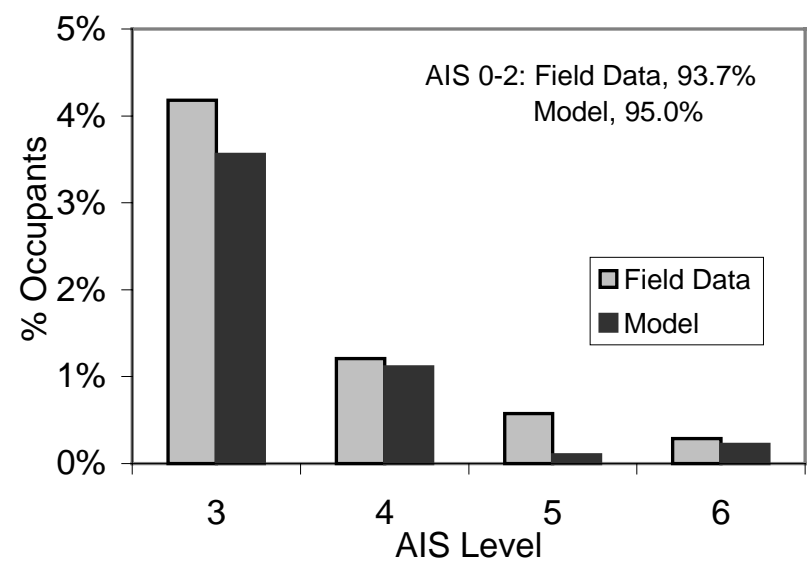

Figure 5. AIS Distribution of Subset Environment Model vs. Field Data Comparison

The model demonstrates very good agreement with field data. The model appears to underestimate AIS 5 injuries by $0.5 \%$ of all occupants, while showing smaller underestimations and generally good agreement in all other AIS categories. 


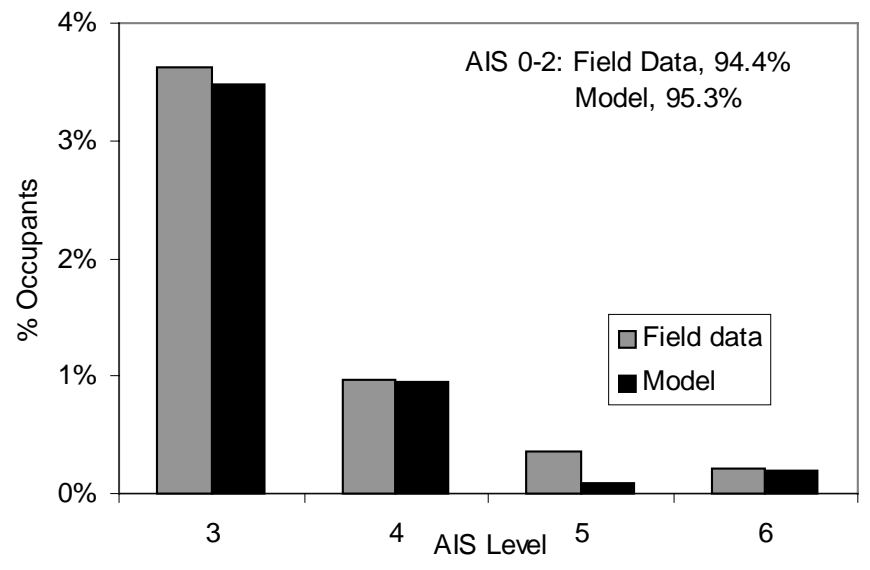

Single-Vehicle Fixed Object Impacts
$n=301,000$ cases/year

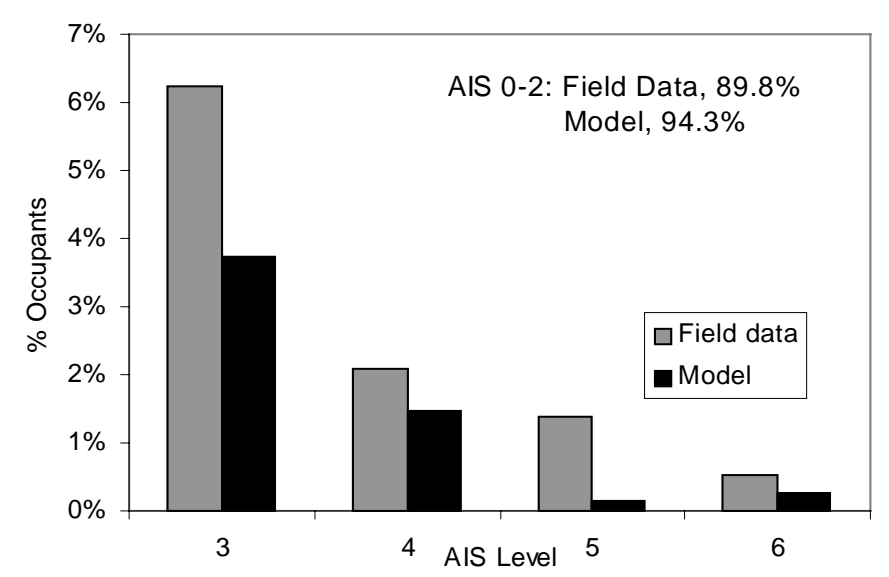

Two-Vehicle Head-on Impacts
$\mathrm{n}=83,000$ cases/year

Figures 6 and 7. AIS Distribution by Accident Mode

In Figures 6 and 7 the model results and field data are presented by accident mode, so that the model's accuracy within each of these groups may be assessed. Figure 6 shows very good agreement with field data among single-vehicle fixed object impacts. Figure 7 shows the model is slightly less accurate for 2-vehicle head-on impacts, underestimating AIS 3 and 5 injuries by $2.5 \%$ and $1.3 \%$, respectively, and underestimating the AIS 4 and 6 categories by smaller amounts.

Although the model shows less accurate prediction of injury distribution for 2-vehicle impacts than single vehicle impacts, the number of cases represented in Figure 7 is also much smaller, at $n=83,000$. Therefore, in terms of the absolute number $n$ of injuries, the model results are roughly equally accurate for single- and two-vehicle impacts. This suggests that larger percentage errors may be acceptable for smaller subsets of data, when absolute numbers of injuries are

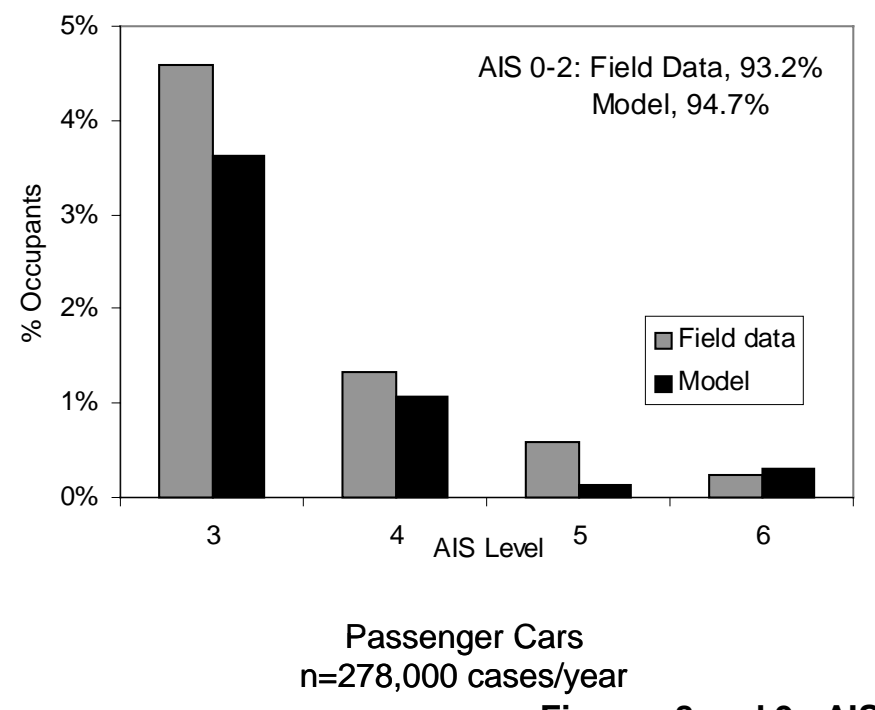

considered.

Figures 8 and 9 show the same data sorted by vehicle type, car and LTV. For car impacts (Figure 8), the model appears to very closely estimate injuries for the AIS 4 and 6 categories. The model underestimates AIS 3 injuries by $1.0 \%$ and AIS 5 injuries by $0.5 \%$ of all car occupants. Among LTV impacts, the model appears to overlook the severest injuries of AIS 5 and 6, while overestimating AIS 3 and 4 injuries by $0.3 \%$ each.

There exist several possible reasons for the underestimation of AIS 5 and 6 injuries in LTVs by the model seen in Figure 9. One source of error may be a systematic tendency within the vehicle or occupant models to predict injuries only up to an artificial maximum threshold. Although these LTV models have been validated at 56kph (35mph), they have been extrapolated to simulate higher speeds of up to $80 \mathrm{kph}(50 \mathrm{mph})$, where the most severe

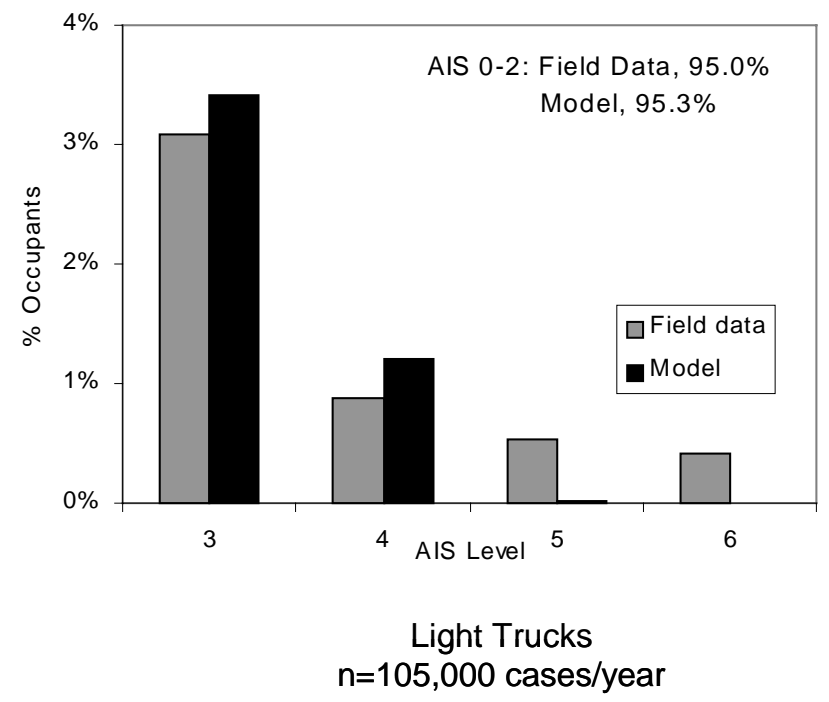

Figures 8 and 9. AIS Distribution by Vehicle Type 
injuries are likely occur. Refinement and validation of the LTV vehicle and occupant models at higher speeds may address this issue, although validation data from high speed crash tests are not currently available for LTVs. Another reason for the underestimation of AIS 5 and 6 injuries among LTV occupants may be that the model assumes air bag availability in all cases, whereas the most severe injury cases reported in field data may occur in non-air bag situations. To eliminate this potential source of error in calculation of severe injuries, future refinements to the model will include simulation of non-air bag cases, or development of approximating functions to estimate injuries in both air bag and non- air bag equipped cases.

Vehicle Aggressivity Analysis. To assess the role of vehicle aggressivity and compatibility within the model environment, serious injury results of only 2 -vehicle impacts between LTVs and cars are shown in Figure 10. This subgroup of cases represents roughly $13 \%$ of the modeled crash environment, or roughly 50,000 occupants, 3,000 of which are seriously injured. The y-axis scale in Figure 10 represents only the percentage of occupants involved in LTVcar impacts (where $100 \%$ reflects 50,000 occupants). The data demonstrates that car occupants undergo significantly more numerous and more severe injuries than their LTV counterparts. AIS 3 injuries in cars outnumber those in LTVs by nearly 2 to 1 , while this ratio for AIS 4 injuries is 3 to 1 . While small numbers of AIS 5 and 6 injuries are predicted for car occupants, virtually no injuries are predicted for LTV occupants in LTV/car collisions. The data shows a clear disadvantage for car occupants, as reflected by simulated crashworthiness behavior in the vehicle and occupant models. For example, the maximum approach speed simulated for 2vehicle collisions is $78 \mathrm{kph}$ per vehicle, or an approach speed of $156 \mathrm{kph}$. Due to the differences in the two vehicles' mass and stiffness, the LTV undergoes a delta-v of only $90 \mathrm{kph}$ (including rebound effects) at this highest impact speed, whereas the car has a much higher delta-v of $107 \mathrm{kph}$. One potential ancillary factor in the lack of AIS 5 and 6 injuries among LTV occupants in LTV/car impacts may be a systematic tendency of the LTV occupant model to

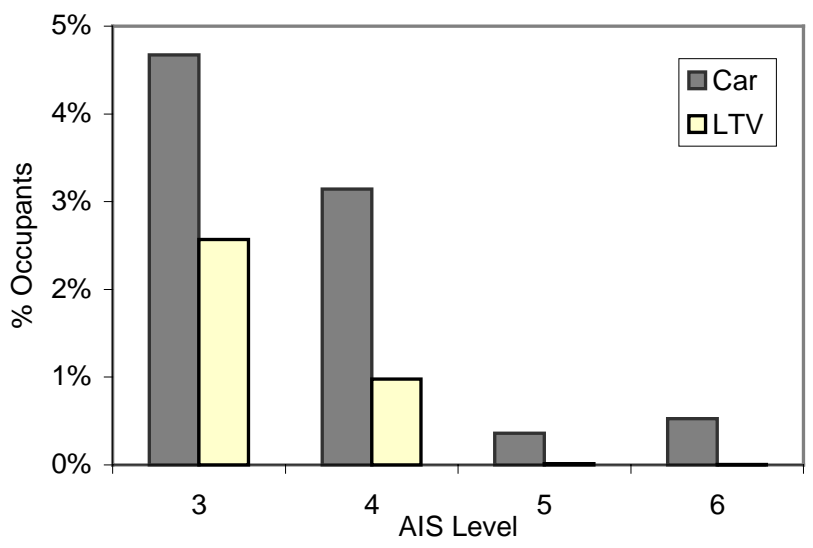

Figure 10. Serious Injuries in LTV/Car Impacts, by Subject Vehicle underpredict injuries, as mentioned in the previous section. Nonetheless, Figure 10 clearly demonstrates a significant increased risk of injury for car occupants in LTV/car collisions.

As LTVs continue to gain popularity among motorists, their potential effect on total safety within the accident environment grows in importance. To study the net safety effects of increasing LTV population within the accident environment (or the frontal impact environment, as the methodology covers here), the model was exercised to assess the sensitivity of occupant injuries as a function of LTV/car fleet mix. Occupant injuries were predicted for the hypothetical cases of a $100 \%$ car fleet and a $100 \%$ LTV fleet, as well as a range of scenarios in between these endpoints, at $10 \%$ intervals. The predicted results for serious injuries are shown in Figure 11. This study assumes that the total number of vehicles in the fleet remains fixed. The results reflect all single- and two-vehicle frontal impacts involving cars and LTVs. For reference, the baseline case presented in Figures $5-10$ represents a fleet consisting of roughly $28 \%$ LTVs, $72 \%$ cars.

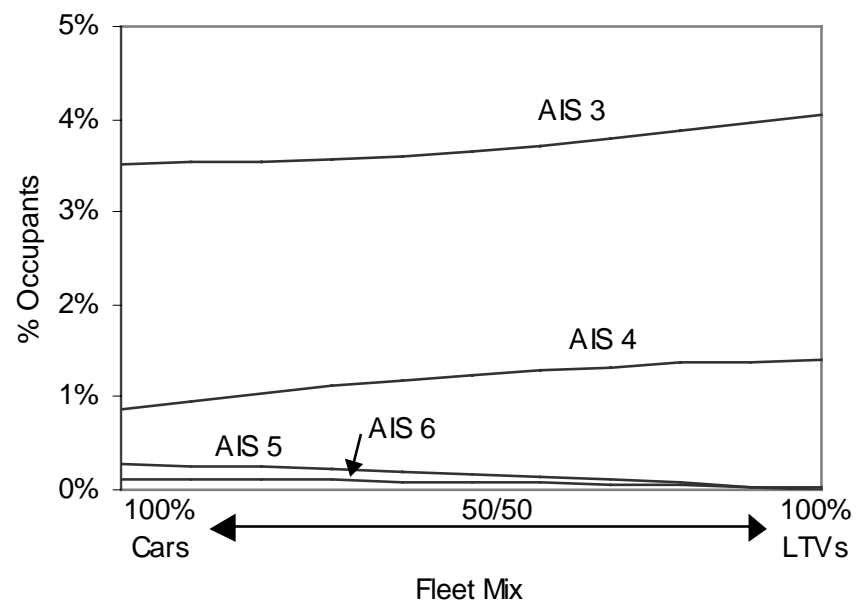

Figure 11. Serious Injuries as a Function of LTV/Car Fleet Mix

The predictive data indicate a net increase in AIS 3 and 4 injuries as the percentage of LTVs climbs, along with a less steep decline in AIS 5 and 6 injuries. The rise in AIS 3 and 4 injuries can be attributed largely to increased injuries in cars during LTV/car impacts, as well as injuries in singlevehicle LTV impacts. The slight drop in (and near elimination of) AIS 5 and 6 injuries is attributed to the decline in cars within the fleet from left to right, as these most severe injuries tend to occur more frequently within cars than LTVs. This is true regardless of crash partner. Because these results include all single- and two- vehicle collisions, cases resulting from single-vehicle and same-vehicle head-on impacts (car-car and LTV-LTV) simply scale up or down as a function of car or LTV population. Figure 11 demonstrates the net result of fleet mix changes within the accident environment, including aggressivity issues in LTV/car impacts as well as same-vehicle and different-vehicle impacts. 


\section{0}

Conclusions

A systems modeling methodology for estimation of harm has been presented and validated for a subset accident environment consisting of single- and two-vehicle frontal impacts. The model has been applied to study the sensitivity of total harm to fleet mix, wherein an incremental increase in LTV population is linked to a rise in moderate to severe injuries.

Components of the methodology include a statistical model providing a probabilistic description of the accident environment, vehicle and occupant models for parametric simulation of crashes, and biomechanical transforms for estimation of injury in each case. The application model presented consists of 504 occupant cases, representing 384,000 drivers and passengers annually, 24,000 of which sustain a serious injury.

The model demonstrates that overall injury trends are very accurately estimated using the system modeling methodology described. The model predicts distribution of AIS level 3 through 6 injuries within $0.5 \%$ of all occupants for each AIS category in the entire subset environment. When validated against field data sorted by accident mode and vehicle type, the model demonstrates very close estimation of injuries, with greater percentage accuracy for single-vehicle collisions than for two-vehicle collisions, and greater percentage accuracy for cars than for LTVs. For nearly all comparisons against field data the model slightly underestimates AIS 3 through 6 injuries. This underestimation may be attributed to several factors built into model assumptions. First, all frontal collisions, including angled and offset frontal collisions, are simulated as full frontal impacts. Hence, some of the more severe injuries resulting from angled and offset impacts that occur in the field may not have been fairly represented by full frontal simulation. Also, the model assumes 100\% air bag availability, when the comparison field data reflects an air bag availability rate of $17 \%$ for the years 1992-97.

Furthermore, injury severity may be slightly underestimated by the fact that compounding effects of combined injuries are not modeled, and injuries to body regions other than the head and chest are not considered.

Observed differences between model results and field data indicate that there exist areas for potential improvement of the application model presented. In addition to validation of the occupant kinematic models in the unbelted case, simulation of non-air bag scenarios would improve the model's results when compared against field data from past years. However, as air bag equipped vehicles eventually dominate the fleet over time, the issue of air bag unavailability will diminish. Further refinement of the statistical model to include more variable joint dependencies and greater resolution across continuous variables such as impact speed may lead to a more accurate prediction of overall harm. The vehicle models used could be improved by representing vehicle geometry, as the 1-dimensional models employed here are adequate for capturing vehicle mass and stiffness behavior, but do not consider geometric effects such as bumper height mismatch. Finally, the implemented biomechanical models, in terms of injury criteria and risk functions for estimating AIS levels from those criteria, are also subject to known limitations.

To study the problem of vehicle aggressivity and compatibility, LTV/car impacts were separately evaluated to identify serious injury trends within each subject vehicle. Car occupants were found to undergo significantly greater harm than their LTV counterparts, by a factor of 2:1 for AIS 3 injuries and 3:1 for AIS 4 injuries. While small numbers of AIS 5 and 6 injuries were predicted for car occupants, these injuries were nearly nonexistent for LTV occupants in LTV/car collisions. Furthermore, the overall sensitivity of total occupant injuries as a function of LTV/car fleet mix was investigated. AIS 3 and 4 injuries were found to rise steadily with LTV population, while AIS 5 and 6 injuries were found to fall less steeply as cars became less prevalent.

Future work. This investigation is an ongoing effort to develop methods for evaluation of fleetwide aggressivity and compatibility in support of NHTSA research initiatives. Further studies will include 3-dimensional lumped parameter or hybrid vehicle models to capture occupant compartment response in angled and offset frontal impacts. The scope of the existing model will be expanded to include side impacts in addition to frontal impacts, and include sensitivity analyses to evaluate the relationship between vehicle crashworthiness in frontal collisions and aggressivity in side impacts. Long term developments include addition of optimization capability to the methodology, to identify optimal vehicle features which lead to a minimization of overall harm.

\section{Acknowledgements}

The authors wish to thank Lawrence Simeone of the Volpe Center for development and simulation of the occupant kinematic models used in this study. They also thank W. Thomas Hollowell and Stephen Summers of NHTSA for support of systems modeling research at the Volpe Center.

\section{References}

Kuchar, Alexandra (2000). A Systems Modeling Methodology for Estimation of Harm in the Automotive Accident Environment. Master's Thesis, Department of Mechanical Engineering, Tufts University.

Eppinger, R., Sun, E., Bandak, F., Haffner, M., Khaewpong, N., Maltese, M., Kuppa, S. Nguyen, T., Takhounts, E., Tannous, R., Zhang, A., and Saul, R. (1999). "Development of Improved Injury Criteria for the Assessment of Advanced Automotive Restraint Systems - II", Supplement to NHTSA Docket No. 1998-4405-9.

Ford Motor Company. (1978). "Safety Systems Optimization Model, Final Report", DOT Report Number DOT HS 601446 . 
Joksch, H. (1998). "Fatality Risks in Collisions Between Cars and Light Trucks", DOT Report Number DOT HS 808802.

Kleinberger, M., Sun, E., Eppinger, R., Kuppa, S. and Saul, R. (1998). "Development of Improved Injury Criteria for the Assessment of Advanced Automotive Restraint Systems", NHTSA Docket No. 1998-4405-9.

Mentzer, S.G. (1999). "The SISAME Program: Structural Crash Model Extraction and Simulation", U.S. Department of Transportation Report (in preparation).

Mentzer, S.G., Radwan, R.A., and Hollowell, W.T. (1992). "The SISAME Methodology for Extraction of Optimal Structural Crash Models", SAE Paper 920358.

National Highway Traffic Safety Administration. (1999). "National Automotive Sampling System: Crashworthiness Data System, 1994-1996", USDOT Report Number DOT HS 808985.

White Jr., K.P., Gabler III, H.C., Pilkey, W.D., and Hollowell, W.T. (1985). "Simulation Optimization of the Crashworthiness of a Passenger Vehicle in Frontal Collisions using Response Surface Methodology", SAE Paper 850512. 